\title{
Expression of long non-coding RNA and mRNA in the hippocampus of mice with type 2 diabetes
}

\author{
LI-YA CI ${ }^{1,2}$, DE-SHAN LIU ${ }^{1}$, JING-QING YANG ${ }^{3}$, YU ZHAO LIU ${ }^{4}$, CHANG LING LI $^{1}$, \\ XI ZHANG ${ }^{1}$, CHUN MEI MA ${ }^{4}$ and RUI TING HU ${ }^{4}$ \\ ${ }^{1}$ Department of Traditional Chinese Medicine, Qilu Hospital of Shandong University, Jinan, Shandong 250012; \\ Departments of ${ }^{2}$ Geriatric Medicine and ${ }^{3}$ Respiratory Medicine, Yantai Affiliated Hospital of \\ Binzhou Medical University, Yantai, Shandong 264100; ${ }^{4}$ Cheeloo College of Medicine, \\ Shandong University, Jinan, Shandong 250012, P.R. China
}

Received October 28, 2017; Accepted August 28, 2018

DOI: $10.3892 / \mathrm{mmr} .2018 .9504$

\begin{abstract}
Long non-coding RNAs (IncRNAs) serve key roles in cell growth, development and various diseases associated with the central nervous system. However, differential expression profiles of lncRNAs in type 2 diabetes have not been reported. The present study aimed to analyze the expression pattern of lncRNA-mRNA in a type 2 diabetic mouse model using microarray analysis. The mouse model of type 2 diabetes was established and the total RNAs were extracted from the hippocampus of the mice used in the present study. The total RNAs were then examined by the GeeDom human lncRNA + mRNA V4.0 expression profile and analyzed through comparing Gene Ontology (GO) enrichment analysis and signal pathway analysis with the Kyoto Encyclopedia of Genes and Genomes (KEGG) database. There were statistically significant differences between the expression of IncRNAs and mRNA in the healthy mice and that of the diabetic mice. In the diabetic mice, 130 different lncRNAs were expressed with 126 significantly upregulated and 4 significantly downregulated and 49 different mRNAs were detected with 45 significantly upregulated and 4 downregulated. GO analysis indicated that the mRNAs that are affected are involved in transport, cell adhesion, ion transport and metabolic processes. KEGG and Reactome enrichment analysis indicated that mRNAs impact on cholinergic synapses, nuclear factor-kB pathway, Toll like receptor 4 cascade and zinc transporter are correlated with cognitive dysfunction in type 2 diabetes. A dynamic lncRNA-mRNA network was constructed containing 123 lncRNAs and 48 mRNAs, which can elucidate the interaction between IncRNA and mRNA.
\end{abstract}

Correspondence to: Dr De-Shan Liu, Department of Traditional Chinese Medicine, Qilu Hospital of Shandong University, 107 Wenhua Xi Road, Jinan, Shandong 250012, P.R. China E-mail: liudeshan@sdu.edu.cn

Key words: long non-coding RNA, mRNA, type 2 diabetes, hippocampus, gene chip
Overall, this is the first study to indicate that lncRNAs are differentially expressed in the type 2 diabetic mice.

\section{Introduction}

Type 2 diabetes mellitus is a highly prevalent disease worldwide and is associated with increased rates of morbidity and mortality. It is estimated that 382 million adults suffered from diabetes in 2013 and that this number will reach 592 million around the world by 2035 (1). It is a metabolic disorder characterized by chronic hyperglycemia. Long-term untreatment or improper treatment may lead to chronic complications of diabetes mellitus, including diabetic nephropathy, diabetic microangiopathy and diabetic neuropathy (2-4). These chronic complications are also important factors, which may cause the patient to succumb to the disease or result in disability.

Long noncoding RNAs (lncRNAs) belong to a heterogeneous class of regulatory ncRNAs with transcript lengths $>200$ nucleotides and serve important roles in gene silencing and posttranscriptional gene regulation $(5,6)$. During recent decades, IncRNAs have been identified as important epigenetic factors in regulating various human diseases, including cancer, neurodegenerative diseases, diabetes and cardiovascular diseases (7-11).

For instance, inhibition of lncRNA insulin-like growth factor 2 (IGF2) antisense RNA promoted angiogenesis in type 2 diabetes through upregulating IGF2 and vascular endothelial growth factor (12). Zhou et al (13) demonstrated that lncRNA metastasis associated lung adenocarcinoma transcript 1 presented an extremely high expression level in pancreatic cancer tissues and cells. It promoted the proliferation of AsPC-1 cells by regulating Hippo-YAP signaling lncRNA HOX transcript antisense RNA and colon cancer associated transcript 2 promoted the proliferation of cervical cancer cells $(14,15)$. A study demonstrated that nine IncRNAs were then combined to form a single prognostic signature for predicting metastatic risk in breast cancer patients (16). Another previous study reported that IncRNA AL049437 may contribute to the risk of Parkinson's disease, while lncRNA AK021630 may reduce the chance of Parkinson's disease (17). In recent years, there 
have been numerous reports of diabetic central nervous system disease (18-20). Roberts et al (21) considered the putative role(s) of IncRNAs in neurodevelopment and brain function with an emphasis on the epigenetic regulation of gene expression. Certain studies demonstrated that the lncRNA TCL1 upstream neural differentiation-associated RNA was demonstrated to be highly conserved among vertebrates and was expressed within the developing nervous system (22) and lncRNA BC200 levels in Brodmann's area 9 [the area of brain affected in Alzheimer's disease (AD)] were demonstrated to be higher in age-matched AD brains compared with normal brains and the relative levels of BC200 RNA in affected areas increased with the severity of AD (23). In addition, the six-lncRNA (AC005013.5, UBE2R2-AS1, ENTPD1-AS1, RP11-89C21.2, AC073115.6 and XLOC_004803) signature may be involved in the immune-associated biological processes and pathways, which are very well known in the context of glioblastoma tumorigenesis (24). Increasing evidence also suggests that long noncoding RNAs serve important regulatory roles in type 2 diabetes.

However, there remains controversy regarding the results of pathophysiology and treatment in cognitive dysfunction of type 2 diabetes. The aim of the present study was to analyze the expression pattern of lncRNAs-mRNA in the hippocampus of type 2 diabetic mice through gene chip detection technology and to investigate novel ideas for identifying abnormal gene expression of cognitive dysfunction and pathophysiology in type 2 diabetes. This study is a preliminary study, using the method of gene chip analysis to investigate possible differences between genes in the diabetic and non-diabetic group. Due to lots of differential gene, further analysis and verification are needed.

\section{Materials and methods}

Mouse model. Twelve C57BL6J female mice (aged 8 weeks old) were bred in a specific pathogen-free laboratory (temperature, $20-24^{\circ} \mathrm{C}$; humidity, 40-70\%) at Shandong University (Jinan, China). Mice were acclimated to the feed for 1 week prior to the initiation of experimental intervention. All mice were housed in standard cages and had free access to food and water. Following this, six mice were randomly selected and labeled as control group. The other 6 mice were fasted for $12 \mathrm{~h}$, then injected with $1 \%$ streptozotocin (Sigma-Aldrich; Merck KGaA, Darmstadt, Germany) solution which was dissolved in $0.05 \mathrm{~mol} / 1$ sodium citrate $(\mathrm{pH} 4.5$; Beyotime Institute of Biotechnology, Haimen, China), at a dose of $60 \mathrm{mg} / \mathrm{kg}$ and injected for 5 days continuously. The control group had intraperitoneal injections of buffer solution at the same dose. After 10 days, the tail vein blood glucose level was detected through a blood glucose meter (Bayer, Newbury, UK). All six mice injected with $1 \%$ streptozotocin solution exhibited high blood glucose levels ( $\geq 16.7 \mathrm{mmol} / \mathrm{l})$ and were considered to be diabetic model mice (25). The control group and the diabetes group were administered daily $10 \mathrm{ml} / \mathrm{kg}$ intragastric normal saline treatments for a total of 12 weeks. Mice were weighed and were subjected to tail vein blood glucose testing once each week.

Following the final saline treatment, the mice were anaesthetized by intraperitoneal injection of $0.8 \%$ pentobarbital
(Sigma-Aldrich; Merck KGaA). The mouse limbs were fixed and the abdomen was exposed, then the abdominal skin, peritoneum and diaphragm were cut to expose the heart. Left ventricular blood was withdrawn through a $1 \mathrm{ml}$ syringe then this injected into a coagulation + separation tube for extraction by $10 \mathrm{~min}$ centrifugation at $900 \mathrm{x} \mathrm{g}$ at $20^{\circ} \mathrm{C}$. Extracted serum was kept frozen at $-80^{\circ} \mathrm{C}$ within a cryopreservation tube. The brain tissue was then obtained and then the hippocampus tissue was isolated and subsequently stored at $-80^{\circ} \mathrm{C}$. The protocol for the present study was approved by the Laboratory Animal Ethics committee of the Qilu Hospital of Shandong University.

Reagents. TRIzol ${ }^{\circledR}$ Reagent (Invitrogen; Thermo Fisher Scientific, Inc., Waltham, MA, USA; cat. no. 15596-026); NucleoSpin ${ }^{\circledR}$ RNA clean-up (cat. no. 740.948.250); Nucleospin ${ }^{\circledR}$ Extract II (cat. no. 740.609.250; both Machery-Nagel, GmbH, Düren, Germany); GeeDom bio-chip universal label reagent (Capital Biotechnology, Co., Ltd., Beijing, China; cat. no. 360069); Cy3-dCTP (GE Healthcare, Chicago, IL, USA; cat. no. PA53031); Cy5-dCTP (GE Healthcare; cat. no. PA55031); 10\% SDS; 20 x sodium citrate buffer (both Capital Biotechnology, Co., Ltd.); Agilent one-color RNA Spike-in kit (cat. no. 5188-5282); Agilent two-color RNA Spike-in kit (cat. no. 5188-5279; both Agilent Technologies Inc., Santa Clara, CA, USA); RNAiso Plus kit (Takara Biotechnology Co., Ltd., Dalian, China); TransScript One-step gDNA Removal and cDNA Synthesis SuperMix kits; TransStart Tip Green qPCR Super MixSYBR; Reverse Transcriptase kit; and TaqMan MGB probes (all Beijing Transgen Biotech Co., Ltd., Beijing, China) were purchased for use in the present study.

Equipment. A 5810R centrifuge was purchased from Eppendorf (Hamburg, Germany); the spectrophotometer ND-1000 was from NanoDrop; Thermo Fisher Scientific, Inc. (Wilmington, DE, USA); the concentrator (instrument model 5301) was from Eppendorf. The Peltier Thermal Cycler PTC-225 (MJ Research, Inc., Waltham, MA, USA). The hybridization Oven (G2545A) was from Agilent Technologies, Inc., as was the Agilent G2565CA Microarray Scanner.

Synthesis and labeling of $c D N A$. Total RNA samples were extracted from three healthy mice (group A) and three type 2 diabetic mice (group B). Total RNA was isolated using TRIzol $^{\circledR}$ reagent (Invitrogen; Thermo Fisher Scientific, Inc.) according to the manufacturer's protocol. The first and second strand cDNA were synthesized with a PrimeScript ${ }^{\mathrm{TM}}$ Double Strand cDNA Synthesis kit according to the manufacturer's protocol (Takara Biotechnology Co., Ltd.). Complementary RNA (cRNA) was transcribed via the T7 Enzyme Mix kit using the second strand cDNA as a template and subsequently purified using NucleoSpin ${ }^{\circledR}$ RNA clean-up (740.948.250; Machery-Nagel, $\mathrm{GmbH}$ ) to eliminate reagents like salt and enzymes according to the manufacturer's protocol. In addition, the aforementioned dNTPs and fluorophores were added to the mixture. Following this, the purified RNA was reverse transcribed using a Reverse Transcriptase kit (Beijing Transgen Biotech Co., Ltd.). 
Table I. Sequences of primers used for reverse transcription-quantitative polymerase chain reaction.

Primer Sequence $\left(5^{\prime}->3^{\prime}\right)$

Prr32 forward

Prr32 reverse

Lmx 1a forward

Lmx 1a reverse

Slc5a7 forward

Slc5a7 reverse

Prr32, proline rich 32; Lmx1a, LIM homeobox transcription factor $1 \alpha$; Slc5a7, solute carrier family 5 member 7 .

IncRNA and mRNA microarray expression profiling. cDNA containing fluorescent labels Cy3-dCTP (GE Healthcare; cat. nos. PA53031) or Cy5-dCTP (GE Healthcare; cat. no. PA55031) or Cy3-dCTP and Cy5-dCTP together were hybridized on the GeeDom human IncRNA+mRNA V4.0 array with GeeDom bio-chip universal label reagent. The microarrays were washed and then scanned using an Agilent G2565CA Microarray Scanner (Agilent Technologies, Inc.) The resulting expression hybridization chip picture was analyzed using Feature Extraction software 10.7 (Agilent Technologies, Inc.). Data normalization and quality control analysis was performed for each sample using GeneSpring GX 11.0 (Agilent Technologies, Inc.). Gene expression differences and P-values were assessed by GeneSpring GX 11.0. Cluster analysis was performed using Cluster 3.0 software (Cluster Software, Inc.). Gene Ontology Enrichment Analysis (GO analysis; http://geneontology.org/) of the target genes, Protein ANalysis THrough Evolutionary Relationships (PANTHER; http://www.pantherdb.org/) and Kyoto Encyclopedia of Genes and Genomes (KEGG; http://www.genome.jp/kegg/pathway.html) pathway analyses, as well as BioCarta (https://cgap.nci.nih. gov/Pathways/BioCarta_Pathways), Pathway Interaction Database (PID; http://pid.nci.nih.gov/), BioCyc (https://biocyc.org/) and Reactome (https://reactome. org/cgi-bin/frontpage?DB=gk_current) analyses, were performed on differential mRNAs. IncRNA-mRNA co-expression analysis was addressed along with target gene prediction and transcription factor prediction for each biological repeat.

Reverse transcription-quantitative polymerase chain reaction $(R T-q P C R)$. Total RNA was isolated using TRIzol ${ }^{\circledR}$ reagent (Invitrogen; Thermo Fisher Scientific, Inc.) according to the manufacturer's protocol. RNA was reverse transcribed to cDNA with TransScript One-step gDNA Removal and cDNA Synthesis SuperMix kits according to the manufacturer's protocol (Transgen Biotechnology Co., Ltd.). RT-qPCR was performed using TransStart Tip Green qPCR Super MixSYBR according to the manufacturer's protocol (Transgen Biotechnology Co., Ltd.). The thermocycling conditions for qPCR were as follows: Initial denaturation at $95^{\circ} \mathrm{C}$ for $30 \mathrm{sec}$, followed by 40 cycles at $95^{\circ} \mathrm{C}$ for $5 \mathrm{sec}, 60^{\circ} \mathrm{C}$ annealing for $30 \mathrm{sec}$. Sequences of primers used for qPCR are presented in Table I. Relative gene expression data was analyzed using the $2^{-\Delta \Delta C q}$ method (26).
Statistical analysis. Statistical analysis was performed using SPSS v20 software (IBM Corp., Armonk, NY, USA). All data are expressed as the mean \pm standard deviation. A T-test indicated consistency between microarray data and RT-qPCR results. $\mathrm{P}<0.05$ was considered to indicate a statistically significant difference. All experiments were repeated in triplicate.

\section{Results}

LncRNA expression. A total of $36,793 \operatorname{lncRNAs}$ were detected within the hippocampus of type 2 diabetic mice. LncRNA and mRNA data revealed differential expression of IncRNA between healthy and diabetic mice captured through data analysis, with differential expression criteria set as fold-change $\geq 2.0$ and $\mathrm{P}<0.05$. Compared with the control mice (group A), diabetic mice (group B) exhibited differential expression of $130 \mathrm{lncRNAs}$, where $126 \mathrm{lncRNAs}$ were demonstrated to be upregulated and 4 lncRNAs were downregulated. The expression levels of IncRNAs were visualized using clustering analysis (Fig. 1A). The differential expression ratios and P-values of IncRNAs in the two groups were also compared using volcano (Fig. 1B) and scatter plots (Fig. 1C). The results revealed a marked difference in the expression levels of lncRNAs exhibited by groups A and B (red points), which suggested that the regulation of IncRNAs is associated with the occurrence and development of type 2 diabetes.

mRNA expression. A total of 30,551 mRNAs were detected within the hippocampus of type 2 diabetic mice. mRNA data was collected from image and data analysis, where the differential expression criteria was set as fold-change $\geq 2.0$ and $\mathrm{P}<0.05$. Compared with group A, group B presented 49 mRNAs with altered expression, where 45 exhibited upregulation and 4 displayed downregulation. The expression levels of mRNAs in groups A and B were visualized using clustering analysis (Fig. 2A). The differential expression ratios and P-values of mRNAs in the two groups were also compared using volcano (Fig. 2B) and scatter plots (Fig. 2C). Fig. 2 demonstrates mRNA differential expression association between different groups.

$R T-q P C R$ validation array. RT-qPCR was performed in order to verify the reliability of microarray data. A total of three differentially expressed mRNAs ( $1 \mathrm{mx} 1 \mathrm{a}$, slc5a7 and prr32) with high expression in the central nervous system were selected from the 49 differentially expressed mRNAs as revealed in Fig. 2A. Alterations in expression levels of $1 \mathrm{mx} 1 \mathrm{a}$ (Fig. 3A), prr32 (Fig. 3B) and slc5a7 (Fig. 3C) revealed by RT-PCR were similar to those observed in microarray data. A T-test indicated consistency between microarray data and RT-qPCR results, and demonstrated that our microarray data is reliable and can be used for bioinformatics analysis in subsequent steps.

Functional analysis of differentially expressed $m R N A$. GO analysis is known as gene enrichment analysis which can be used to confirm gene and gene production associated bio-processes, cellular composition and molecular functions. 

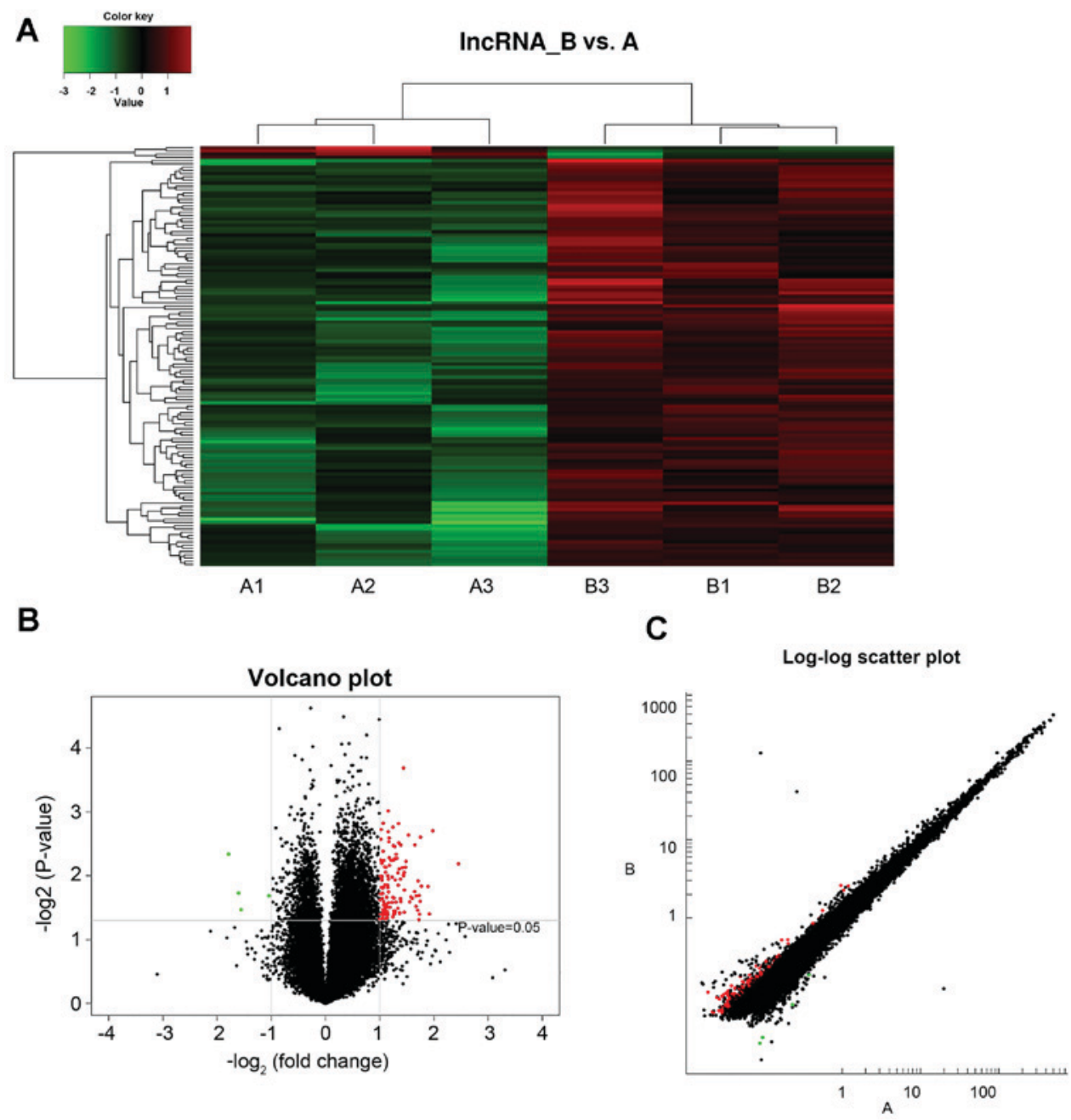

Figure 1. Differential expression of lncRNA by (A) cluster analysis, (B) volcanic map and (C) scatter plot in healthy and type 2 diabetic mice. Red and green dots represent upregulated and downregulated lncRNAs, respectively, lnc, long non-coding.

GO and KEGG analyses can be used to further evaluate the function of significantly expressing trend genes. As presented in Fig. 4, GO enrichment analysis (identification of the biological processes involved in the enrichment of transcripts) indicated that cognitive dysfunction in type 2 diabetes was correlated with transport, cell adhesion, ion transport, metabolic processes and other important biological processes. In addition, KEGG and Reactome enrichment analysis, which were performed to validate important module functions, demonstrated that the pathology of cognitive dysfunction in type 2 diabetes is associated with the regulation of cholinergic synapses, the NF-kB pathway, the Toll like receptor 4 (TLR4) cascade, and the zinc transporter (Fig. 5).

LncRNA-mRNA network. In order to identify important molecular mechanisms of lncRNA associated with cognitive dysfunction in type 2 diabetes, a dynamic lncRNA-mRNA network (Fig. 6) was constructed, which contained 123 lncRNA and 48 mRNA. To address interactions between genes, a threshold of $\geq 0.997$ was implemented to evaluate the coexpression of 1ncRNA and mRNA via quantization of the degree and the size of the circle. From the figure, crucial lncRNAs were demonstrated, which may serve an important role in the pathology of type 2 diabetes, including lncRNA ENSMUST00000188753, lncRNA gi/755552777/ref/XR_875577.1/ and lncRNA gi/755494846/ref/XR_387234.2/. In this figure, the size of a circle indicates the ability of a gene to interact according to its degree of quantification.

A co-expression network based on differential lncRNAs and mRNA of healthy and type 2 diabetic mice is presented. Green nodes represent mRNAs and yellow nodes represent lncRNAs. Red lines express positive correlation and blue lines express negative correlation.

\section{Discussion}

The present study is the first to the best of our knowledge to report on IncRNA expression profiles in the hippocampus of type 2 diabetic mice. Cognitive dysfunction in type 2 diabetes is a complex biological process involving a number of molecular and cellular events. Although lncRNA was once regarded as genome 'noise', IncRNAs have been demonstrated to be enriched in the central nervous system and have important implications for growth and development (27). Therefore, understanding the lncRNA expression profile is critical for investigating the potential function of lncRNAs in cognitive dysfunction in type 2 diabetes mellitus. 


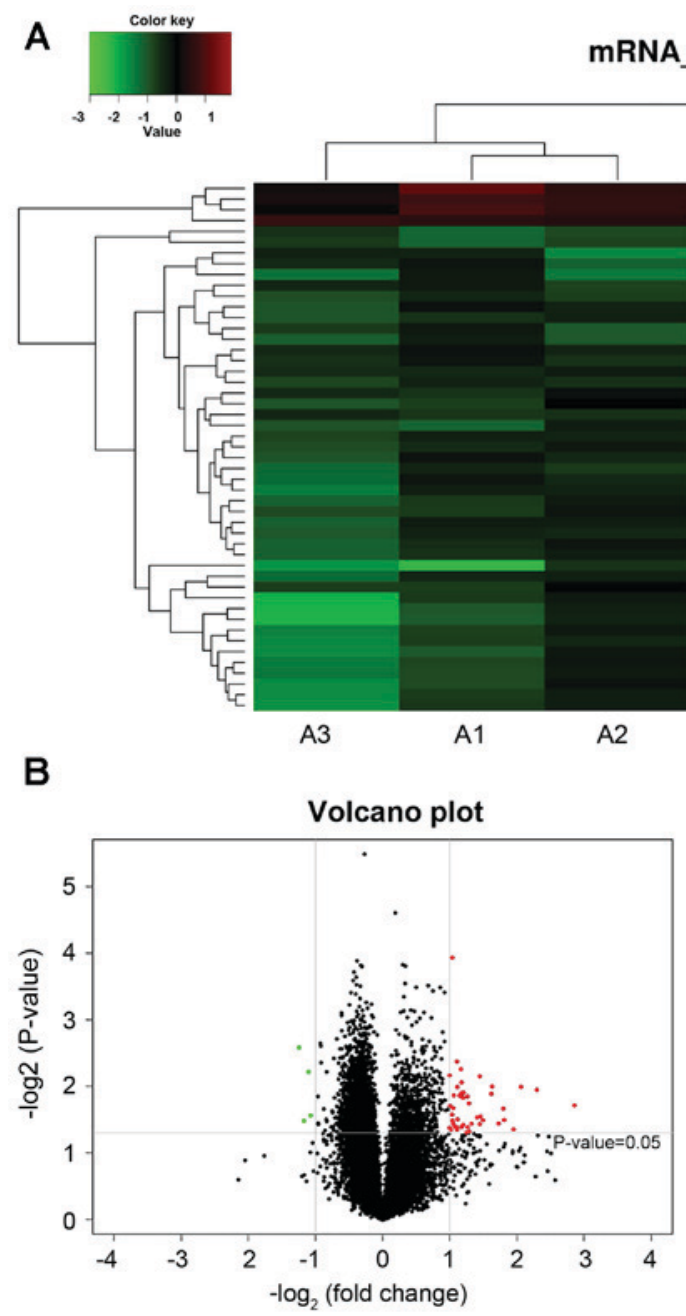

RNA_Bvs. A 


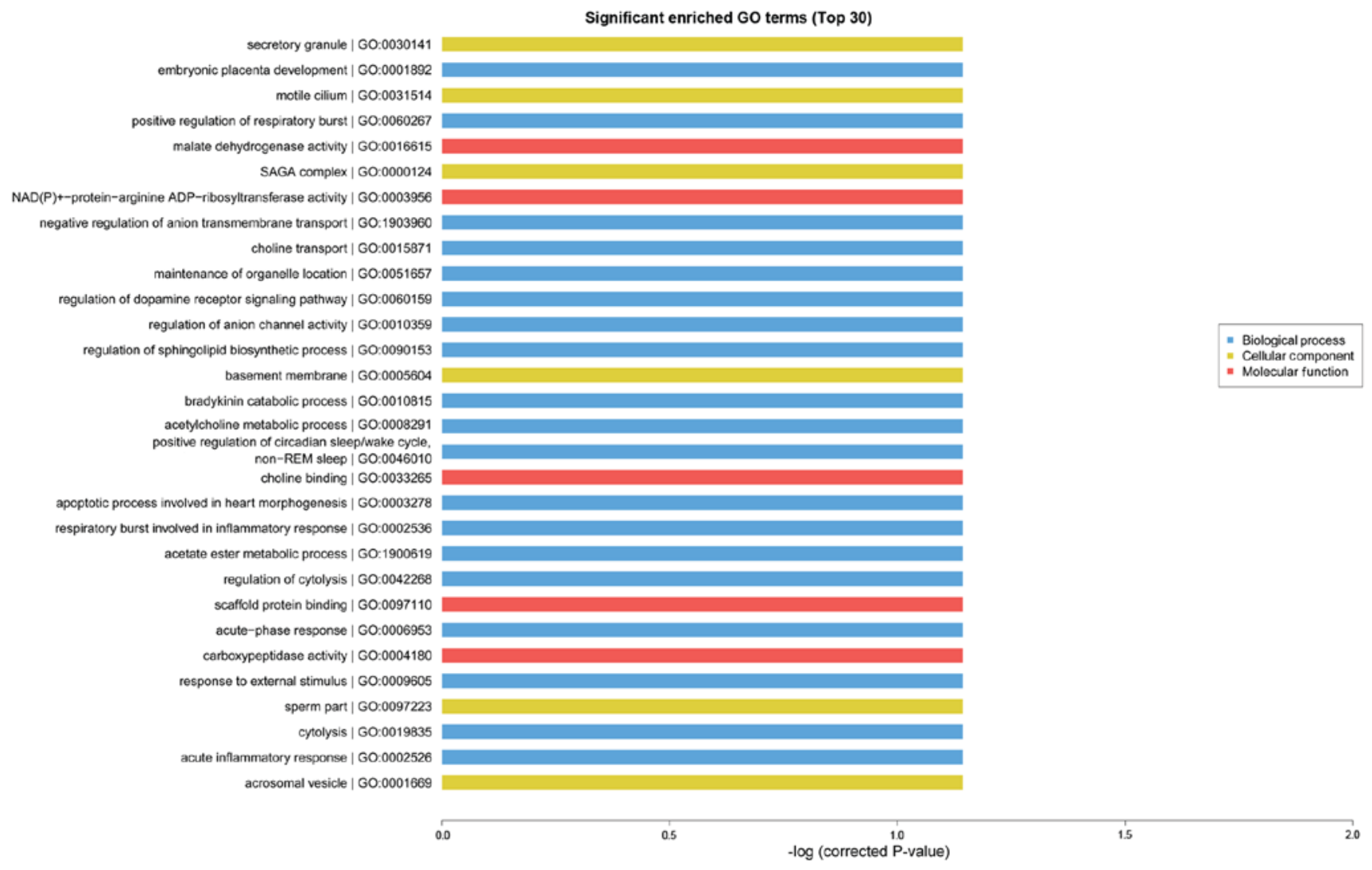

Figure 4. GO functional analysis of significant enrichment in healthy and type 2 diabetic mice. GO, Gene Ontology.

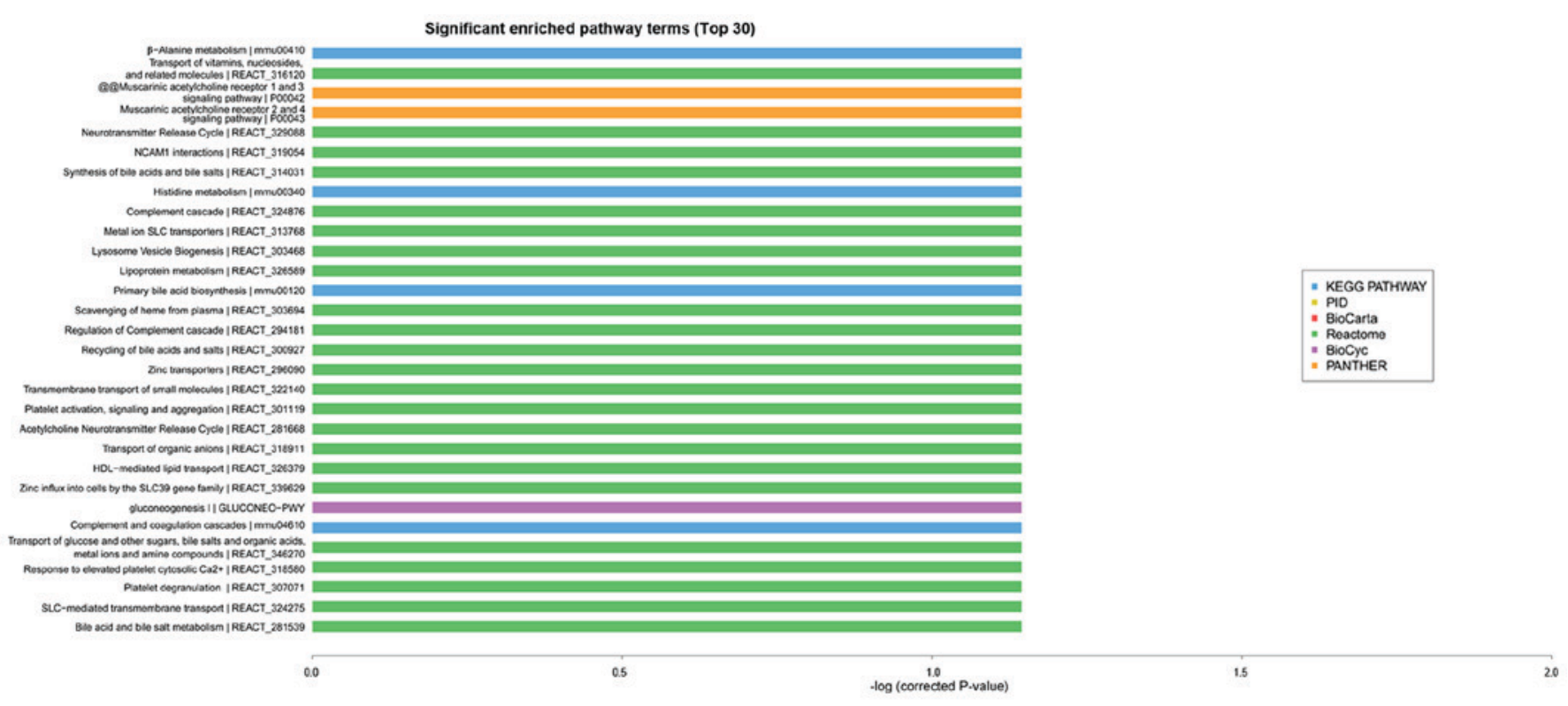

Figure 5. Pathway analysis of significant enrichment in healthy and type 2 diabetic mice. KEGG, Kyoto Encyclopedia of Genes and Genomes; PANTHER, Protein ANalysis THrough Evolutionary Relationships; PID, Pathway Interaction Database.

Animal models serve an important role in the study of type 2 diabetes. A reliable animal model was selected for the extraction of whole cell RNA from the hippocampus of 3 animals in each treatment condition. Experiments were conducted on triplicate samples to ensure reliable and accurate microarray results were associated with RT-qPCR results. After the microarray results were determined to be reliable,
RNA with a significant change in the level of expression (multiples change $\geq 2$ ) was selected for bioinformatics analysis.

According to significantly altered mRNA expression, potential LncRNA functions were studied through GO enrichment and pathway analysis. GO analysis indicated altered expression in genes involved in transport, cell adhesion, ion transport and metabolic processes. KEGG and Reactome 


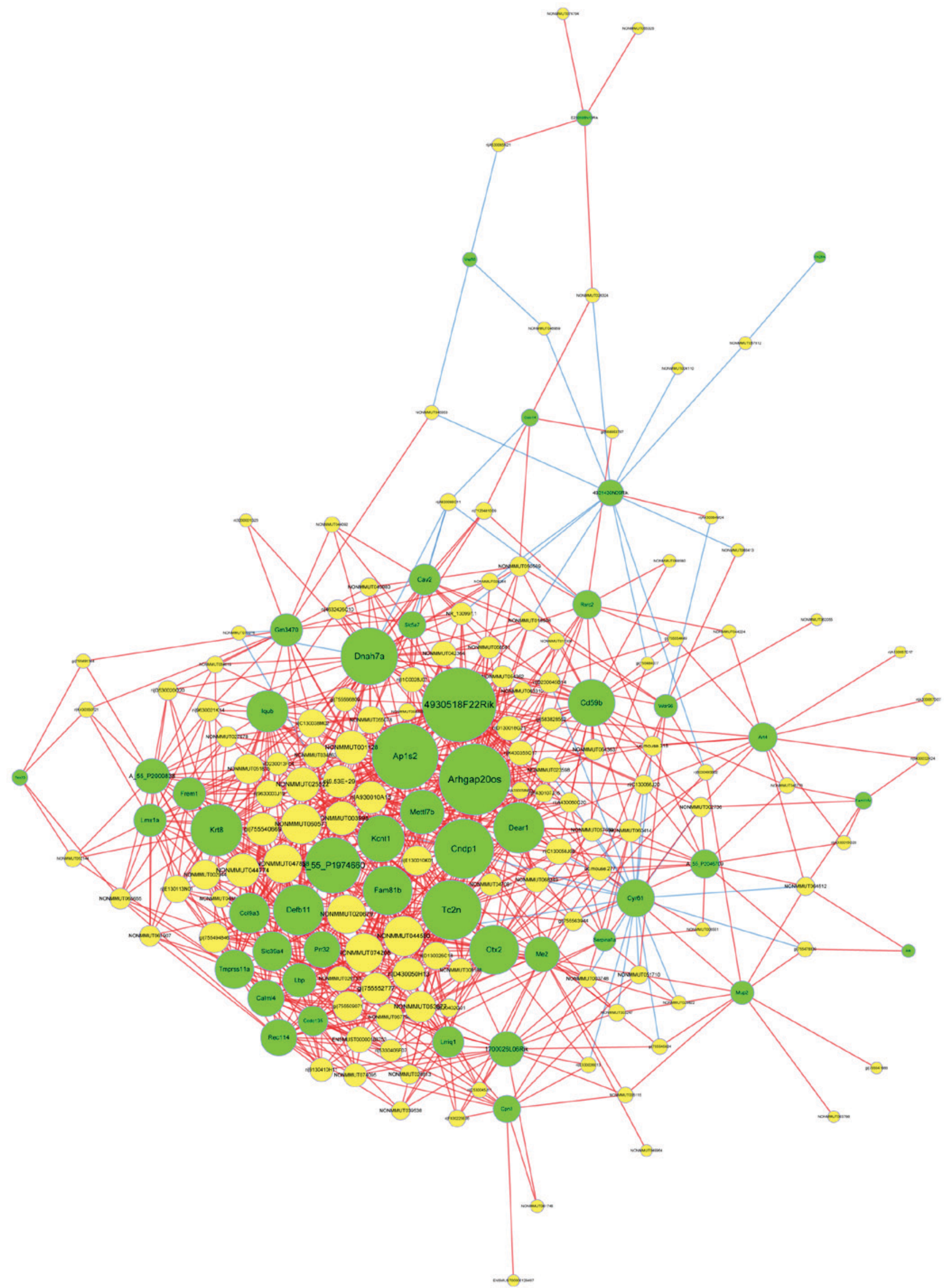

Figure 6. Coding-non-coding gene co-expression network. Co-expression network based on differential lncRNAs and mRNA of healthy and type 2 diabetic mice. Green nodes represent mRNAs and yellow nodes represent lncRNAs. Red lines indicate positive correlation and blue lines indicate negative correlation. Lnc, long noncoding. 
enrichment analysis indicated that functionality of cholinergic synapses, the NF-kB pathway, TLR4 cascade and zinc transporter are associated with the pathological alterations of cognitive dysfunction with type 2 diabetes. Constructing lncRNA-mRNA network improved understanding of the possible interactions and associations between differentially expressed lncRNAs and mRNAs.

The cholinergic synaptic pathway is a classical signaling pathway, which is involved in the synthesis and release of acetylcholine. It is an important pathway for the transmission of information in the nervous system. A previous study demonstrated that cholinergic pathway damage is one of the mechanisms leading to cognitive dysfunction in type 2 diabetes (28). Animal experiments demonstrated decreased cholinergic system activity in the brain tissue of diabetic rats and significantly decreased cholinergic fiber density in the hippocampal CA1 region and molecular layer of the dentate gyrus (29). Cognitive function can be affected by damage of cholinergic nerves due to inflammation and oxidative stress (29). A previous study indicated that hypomnesia of zebra fish caused by hyperglycemia exhibits a close association with acetylcholinesterase activity elevation and galanthamine can help with hypomnesia (30). The above results suggested that the cholinergic synaptic pathway serves an important role in the cognitive function of type 2 diabetic mice. Iwamoto et al (31) demonstrated the gene solute carrier family 5 member 7 (SLC5A7) has a high affinity for the transport of acetylcholine in cholinergic nerve terminals. In the present study the expression level of SLC5A7 mRNA was upregulated and was significantly associated with one lncRNA (gil755552777|refIXR_875577.1I) in the network, the two were highly expressed. Therefore it was concluded that gil755552777/refIXR_875577.1I may regulate the transport of acetylcholine through upregulation of SLC5A7 expression and then affect the expression of cognitive function, which provides us with ideas for further research.

Additionally, there are a number of other signaling pathways that can affect cell proliferation, cell differentiation, gene expression regulation and cell survival. One of them is the NF- $\kappa \mathrm{B}$ signaling pathway. As an essential nuclear factor, $\mathrm{NF}-\kappa \mathrm{B}$ participates in pathophysiological processes including inflammation and apoptosis. In quiescent cells, NF- $\kappa \mathrm{B}$ can combine with inhibitor- $\kappa \mathrm{B}(\mathrm{I} \kappa \mathrm{B})$ to form a tripolymer in the cytoplasm, with no genetic transcription activity. When cells are stimulated by proinflammatory cytokines, hyperglycemia, oxidative stress and ultraviolet radiation IкB- $\alpha$ is phosphorylated and degraded, and then NF- $\kappa \mathrm{B}$ is subsequently released into the nucleus to combine with the specific site of a nuclear target gene, thereby promoting the expression of inflammation associated genes including cytokines, adhesion molecules, chemotactic factor, which serve an important role in diabetes and associated chronic complications (32). A previous study (33) hypothesized that inflammatory cytokines could induce apoptosis pathways, regulate the apoptosis of mouse pancreatic $\beta$-cell factor, destroy the viability and integrity of the islets and lead to lack of insulin secretion by activating NF- $\kappa \mathrm{B}$. NF- $\kappa \mathrm{B}$ sustained activation and inflammation associated expression of cytokines, adhesion molecules, chemotactic factors exists the hippocampus of diabetic mice treated with streptozotocin, which can lead to cerebrovascular trauma and nerve cell apoptosis (34). A study reported that IncRNA-UCA1 dynamically regulates $\mathrm{NF}-\kappa \mathrm{B}$ in the hippocampus of epilepsy rats (35). In addition, the $N F-\kappa B$ signaling pathway mediated by TLR4 serves a key role in the activation and regulation of inflammatory responses in brain tissues damaged by intracerebral hemorrhage (36). Previous studies have demonstrated that Lap is associated with $\mathrm{NF}-\kappa \mathrm{B}$, and $\mathrm{NF}-\kappa \mathrm{B}$ is associated with changes in hippocampal function during diabetes mellitus. Therefore, our future studies will investigate whether Lap affects cognitive impairment in diabetic hippocampal tissues. In the present experiment the expression level of this mRNA was upregulated and was significantly associated with lncRNA gi/755552777/ref/XR_875577.1/ and IncRNA gi/755494846/ref/XR_387234.2/ in the network, which were both highly expressed. Therefore, in future studies we aim to investigate the hypothesis that lncRNA gi/755552777/ref/XR_875577.1/ and 1ncRNA gi/755494846/ref/XR_387234.2/ can activate the NF- $\kappa$ B pathway through upregulation of Lap expression, promote the inflammatory process in the hippocampus, and then affect the expression of cognitive function.

However, the present study also has certain limitations. For example, data reliability is limited due to the small sample size for the microarrays. In addition, with respect to the research methods used, only differentially expressed lncRNA functions can be predicted, but it is not possible to determine how these lncRNA regulate target gene expression. The IncRNA function can be validated by overexpression and RNA interference. Further experiments are needed. There remain certain shortcomings in the data analysis, such as the use of a small sample size. Furthermore, genes with significantly different expression will be verified by PCR and western blotting. The aim of future studies will be to further study and pay attention to the molecular mechanism of lncRNA. Further ingenuity pathway analyses and network analyses are needed to provide more information about biological relevance and the significance of the identified genes/pathways.

In conclusion, the present study demonstrated differential expression profiles of IncRNA in the hippocampal tissue of type 2 diabetes, which will help to study the pathophysiology of cognitive impairment in type 2 diabetes.

\section{Acknowledgements}

The authors would like to thank Dr.Aubryanna Hettingtonhouse of New York University for checking the manuscript.

\section{Funding}

The present study was partly supported by the National Natural Science Foundation of China (grant no. 81173250) and the grants from the Project of Natural Foundation of Shandong Province, China (grant no. ZR2017MH039).

\section{Availability of data and materials}

The datasets used and/or analyzed during the current study are available from the corresponding author on reasonable request. 


\section{Authors' contributions}

L-YC, YZL and CLL established the diabetic mice model. L-YC, J-QY, XZ and CMM performed the experiments. L-YC, D-SL and J-QY conceived the experimental design. L-YC, D-SL and RTH analyzed the data and wrote the manuscript. All authors read and approved the final manuscript.

\section{Ethics approval and consent to participate}

The protocol for the present study was approved by the Laboratory Animal Ethics committee of the Qilu Hospital of Shandong University (Jinan, China).

\section{Patient consent for publication}

Not applicable.

\section{Competing interests}

The authors declare that they have no competing interests.

\section{References}

1. Guariguata L, Whiting DR, Hambleton I, Beagley J, Linnenkamp U and Shaw JE: Global estimates of diabetes prevalence for 2013 and projections for 2035. Diabetes Res Clin Pract 103: 137-149, 2014.

2. Agarwal SK, Saikia UK, Sarma D and Devi R: Assessment of glomerular and tubular function in the evaluation of diabetic nephropathy: A cross-sectional study. Indian J Endocrinol Metab 22: 451-456, 2018

3. Fi Z, Kovács G and Szentes V: Role of trimetazidine in the treatment of diabetic microangiopathy in ischaemic heart disease Ore Hetil 156: 765-768, 2015 (In Hungarian).

4. De Gregorio C, Contador D, Campero M, Ezquer M and Ezquer F: Characterization of diabetic neuropathy progression in a mouse model of type 2 diabetes mellitus. Biol Open 7 : bio036830, 2018.

5. ENCODE Project Consortium: An integrated encyclopedia of DNA elements in the human genome. Nature 489: 57-74, 2012.

6. Mattick JS: RNA regulation: A new genetics? Nat Rev Genetics 5: 316-323, 2004.

7. Kataoka M and Wang DZ: Non-coding RNAs including miRNAs and lncRNAs in cardiovascular biology and disease. Cells 3: 883-898, 2014

8. Liu JY, Yao J, Li XM, Song YC, Wang XQ, Li YJ, Yan B and Jiang Q: Pathogenic role of lncRNA-MALAT1 in endothelial cell dysfunction in diabetes mellitus. Cell Death Dis 5: e1506, 2014.

9. Ng SY, Lin L, Soh BS and Stanton LW: Long noncoding RNAs in development and disease of the central nervous system. Trends Genet 29: 461-468, 2013

10. Lalevee S and Feil R: Long noncoding RNAs in human disease: Emerging mechanisms and therapeutic strategies. Epigenomics 7 : $877-879,2015$.

11. Prensner JR and Chinnaiyan AM: The emergence of lncRNAs in cancer biology. Cancer Discov 1: 391-407, 2011.

12. Zhao Z, Liu B, Li B, Song C, Diao H, Guo Z, Li Z and Zhang J: Inhibition of long noncoding RNA IGF2AS promotes angiogenesis in type 2 diabetes. Biomed Pharmacother 92: 445-450, 2017.

13. Zhou Y, Shan T, Ding W, Hua Z, Shen Y, Lu Z, Chen B and Dai T: Study on mechanism about long noncoding RNA MALAT1 affecting pancreatic cancer by regulating Hippo-YAP signaling. J Cell Physiol 233: 5805-5814, 2018.

14. Wu L, Jin L, Zhang W and Zhang L: Roles of long non-coding RNA CCAT2 in cervical cancer cell growth and apoptosis. Med Sci Monit 22: 875-879, 2016.
15. Sun J, Chu H, Ji J, Huo G, Song Q and Zhang X: Long non-coding RNA HOTAIR modulates HLA-G expression by absorbing miR-148a in human cervical cancer. Int J Oncol 49: 943-952, 2016.

16. Sun J, Chen X, Wang Z, Guo M, Shi H, Wang X, Cheng L and Zhou M: A potential prognostic long non-coding RNA signature to predict metastasis-free survival of breast cancer patients. Sci Rep 5: 16553, 2015.

17. Ni Y, Huang H, Chen Y, Cao M, Zhou H and Zhang Y: Investigation of long non-coding RNA expression profiles in the substantia nigra of parkinson's disease. Cell Mol Neurobiol 37: 329-338, 2017.

18. Chung JY, Kim HS and Song J: Iron metabolism in diabetes-induced Alzheimer's disease: A focus on insulin resistance in the brain. Biometals 31: 705-714, 2018.

19. Giatti S, Diviccaro S and Melcangi RC: Neuroactive steroids and sex-dimorphic nervous damage induced by diabetes mellitus. Cell Mol Neurobiol, 2018 (Epub ahead of print).

20. Zhai Y, Meng X, Ye T, Xie W, Sun G and Sun X: Inhibiting the NLRP3 inflammasome activation with MCC950 ameliorates diabetic encephalopathy in db/db mice. Molecules 23: 522, 2018.

21. Roberts TC, Morris KV and Wood MJ: The role of long non-coding RNAs in neurodevelopment, brain function and neurological disease. Philos Trans R Soc Lond B Biol Sci 369: 20130507, 2014.

22. Lin N, Chang KY, Li Z, Gates K, Rana ZA, Dang J, Zhang D, Han T, Yang CS, Cunningham TJ, et al: An evolutionarily conserved long noncoding RNA TUNA controls pluripotency and neural lineage commitment. Mol Cell 53: 1005-1019, 2014.

23. Mus E, Hof PR and Tiedge H: Dendritic BC200 RNA in aging and in Alzheimer's disease. Proc Natl Acad Sci USA 104: 10679-10684, 2007.

24. Zhou M, Zhang Z, Zhao H, Bao S, Cheng L and Sun J: An immune-related six-lncRNA signature to improve prognosis prediction of glioblastoma multiforme. Mol Neurobiol 55: 3684-3697, 2018

25. Heineke EW, Johnson MB, Dillberger JE and Robinson KM: Antioxidant MDL 29,311 prevents diabetes in nonobese diabetic and multiple low-dose STZ-injected mice. Diabetes 42: 1721-1730, 1993.

26. Livaka KJ and Schmittgen TD: Analysis of relative gene expression data using real-time quantitative PCR and the 2(-Delta Delta C(T)) method. Methods 25: 402-408, 2001.

27. Guennewig B and Cooper AA: The central role of noncoding RNA in the brain. Int Rev Neurobiol 116: 153-194, 2014.

28. Erkinjuntti T, Roman G, Gauthier S, Feldman H and Rockwood K: Emerging therapies for vascular dementia and vascular cognitive impairment. Stroke 35: 1010-1017, 2004.

29. Kuhad A and Chopra K: Effect of sesamol on diabetes-associated cognitive decline in rats. Exp Brain Res 185: 411-420, 2008.

30. Capiotti KM, De Moraes DA, Menezes FP, Kist LW, Bogo MR and Da Silva RS: Hyperglycemia induces memory impairment linked to increased acetylcholinesterase activity in zebrafish (Danio rerio). Behav Brain Res 274: 319-325, 2014.

31. Iwamoto H, Calcutt MW and Blakely RD: Differential impact of genetically modulated choline transporter expression on the release of endogenous versus newly synthesized acetylcholine. Neurochem Int 98: 138-145, 2016.

32. Hao X, Ma B, Song G, Ye W, Li W and Tang Y: The role of inflammatory factor NF-kappa B in the pathogenesis and treatment of type 2 diabetes mellitus. Hebei Med J 32 97-99, 2010 (In Chinese).

33. Park E, Lee SM, Lee JE and Kim JH: Anti-inflammatory activity of mulberry leaf extract through inhibition of NF- $\mathrm{B}$. J Funct Foods 5: 178-186, 2013.

34. Yao X and Jin X: NF- $\kappa$ B in expression of hippocampal neurons with chronic diabetic rats. Chin J Med Pract 4: 512, 2005 (In Chinese).

35. Wang HK, Yan $\mathrm{H}$, Wang $\mathrm{K}$ and Wang J: Dynamic regulation effect of long non-coding RNA-UCA1 on NF- $\kappa$ B in hippocampus of epilepsy rats. Eur Rev Med Pharmacol Sci 21: 3113-3119, 2017.

36. Peng Q, Liu H, Shi S and Li M: Lycium ruthenicum polysaccharide attenuates inflammation through inhibiting TLR4/NF- $\mathrm{BB}$ signaling pathway. Int J Biol Macromol 67: 330-335, 2014. 